\title{
Performance of the ATLAS Hadronic Tile Calorimeter in Run-2 and its Upgrade for the High Luminosity LHC
}

\author{
Oleg Solovyanov ${ }^{1, \star}$ on behalf of the ATLAS Collaboration \\ ${ }^{1}$ State Research Center Institute for High Energy Physics (Protvino), NRC KI
}

\begin{abstract}
The Tile Calorimeter (TileCal) of the ATLAS experiment at the LHC is the central hadronic calorimeter designed for energy reconstruction of hadrons, jets, tauparticles and missing transverse energy. TileCal is a scintillator-steel sampling calorimeter and it covers the region of pseudo-rapidity up to 1.7, with almost 10000 channels measuring energies ranging from $\sim 30 \mathrm{MeV}$ to $\sim 2 \mathrm{TeV}$. Each stage of the signal production, from scintillation light to the signal reconstruction, is monitored and calibrated.
\end{abstract}

The performance of the Tile calorimeter has been studied in-situ employing cosmic ray muons and a large sample of proton-proton collisions, acquired during the operations of the LHC. Prompt isolated muons of high momentum from electroweak bosons decays are employed to study the energy response of the calorimeter at the electromagnetic scale. The calorimeter response to hadronic particles is evaluated with a sample of isolated hadrons. The modelling of the response by the Monte Carlo simulation is discussed. The calorimeter timing calibration and resolutions are studied with a sample of multijets events. Results on the calorimeter operation and performance are presented, including the calibration, stability, absolute energy scale, uniformity and time resolution. TileCal performance satisfies the design requirements and has provided an essential contribution to physics results in ATLAS.

The Large Hadron Collider (LHC) has envisaged a series of upgrades towards a High Luminosity LHC (HL-LHC), delivering five times the LHC nominal instantaneous luminosity. The ATLAS Phase II upgrade, in 2024, will accommodate the detector and data acquisition system for the HL-LHC. In particular, the Tile Calorimeter will undergo a major replacement of its on- and off-detector electronics. All signals will be digitised and then transferred directly to the off-detector electronics, where the signals will be reconstructed, stored, and sent to the first level of trigger at a rate of $40 \mathrm{MHz}$. This will provide better precision for the calorimeter signals used by the trigger system and will allow the development of more complex trigger algorithms. Changes to the electronics will also contribute to the reliability and redundancy of the system. Three different front-end options are presently being investigated for the upgrade. Results of extensive laboratory tests and with beams of the three options will be presented, as well as the latest results on the development of the power distribution and the off-detector electronics.

^e-mail: Oleg.Solovyanov@ihep.ru 

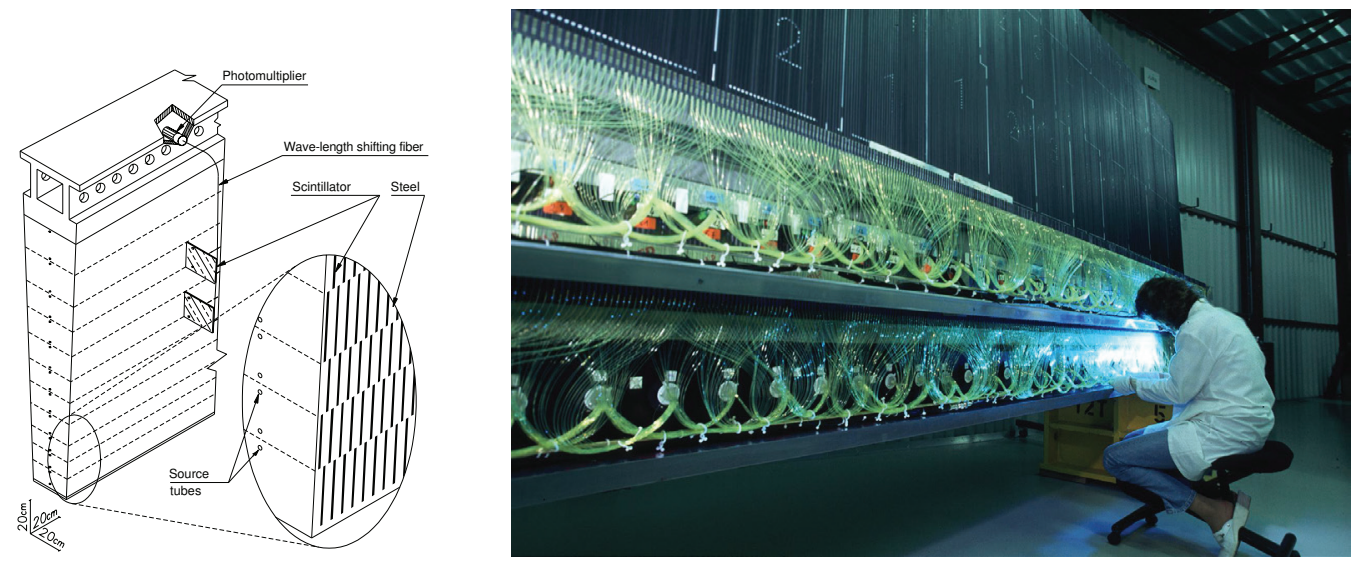

Figure 1. Tile Calorimeter module design and a picture of a long barrel module during instrumentation [2]. Copyright 2017 CERN for the benefit of the ATLAS Collaboration. CC-BY-4.0 license.

\section{Introduction}

In high energy physics experiments, calorimeters are essential for measurements of particle properties, and the hadronic calorimeters, in particular, are crucial in measuring jets, missing transverse energy, taus and muons, and in providing triggers at all levels.

One of the Large Hadron Collider (LHC) general purpose experiments, ATLAS [1], contains hadronic calorimeter, called Tile Calorimeter (TileCal) [2]. Tile TileCal is a non-compensating sampling calorimeter, with steel as a radiator and scintillating tiles as an active medium. The $3 \mathrm{~mm}$ thick tiles are composed of doped polystyrene, and are oriented perpendicular to the beam axis. The light from the tiles is read out via wavelength shifting (WLS) fibres, connected to both short edges of the tiles. The WLS fibres are bundled and read out by photomultiplier tubes, located in a module's girder, as shown in Fig. 1. The calorimeter is divided into 3 cylinders: one Long Barrel (LB) and two Extended Barrels (EB). Each cylinder is further subdivided azimuthally in 64 modules. The calorimeter is $12 \mathrm{~m}$ in overall length with a $4.25 \mathrm{~m}$ outer radius.

The Long Barrel module covers the pseudo-rapidity region $|\eta|<1.0$, while the Extended Barrels cover $0.8<|\eta|<1.7$. Each module have three longitudinal layers (A,BC,D), with a total thickness in radiation lengths of about 7.4 $\lambda$. The calorimeter cells are defined by the WLS fibre routing, providing about 5200 cells in the entire calorimeter with $0.1 \times 0.1 \Delta \eta \times \Delta \phi$ granularity for A and BC cells, and $0.2 \times 0.1 \Delta \eta \times \Delta \phi$ for the outer D cells. Analogue trigger sums from pseudo-projective towers are provided for the first level trigger. The dynamic range of the PMTs and associated electronics, from $10 \mathrm{MeV}$ to $750 \mathrm{GeV}$, allows for the measurements of single particles and large jets. The design resolution for jets is $50 \% / \sqrt{E} \oplus 3 \%$ and the response is linear within $2 \%$ for up to $4 \mathrm{TeV}$ jets [2].

The readout is organised as follows. The PMT signals are shaped and amplified with two gains (1:64 ratio); then both gains are digitised in parallel by $40 \mathrm{MHz} 10$ bits sampling ADCs. The digitised samples are temporarily stored in pipeline memory, until the arrival of first level trigger, when the data of one of the gains are transferred to the back-end electronics via optical links, as shown in Fig. 2.

The signal is reconstructed from 7 samples by applying an optimal filtering (OF) algorithm and the energy, time and quality factors are extracted from signal samples, with the amplitude of the signal proportional to the deposited energy (shaping). To minimise noise, the OF algorithm is using 


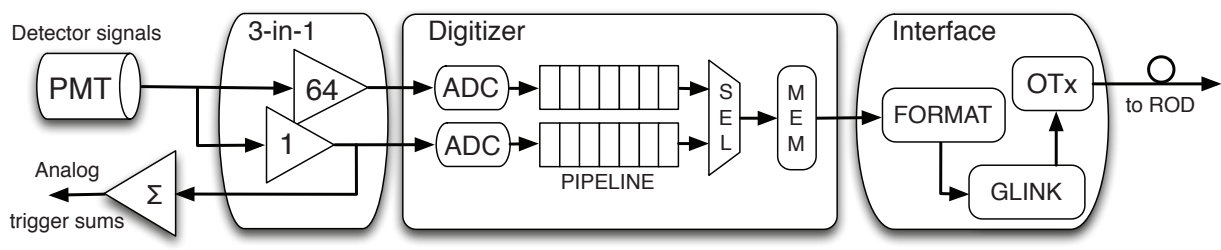

Figure 2. Tile Calorimeter readout scheme [2]. Copyright 2017 CERN for the benefit of the ATLAS Collaboration. CC-BY-4.0 license.

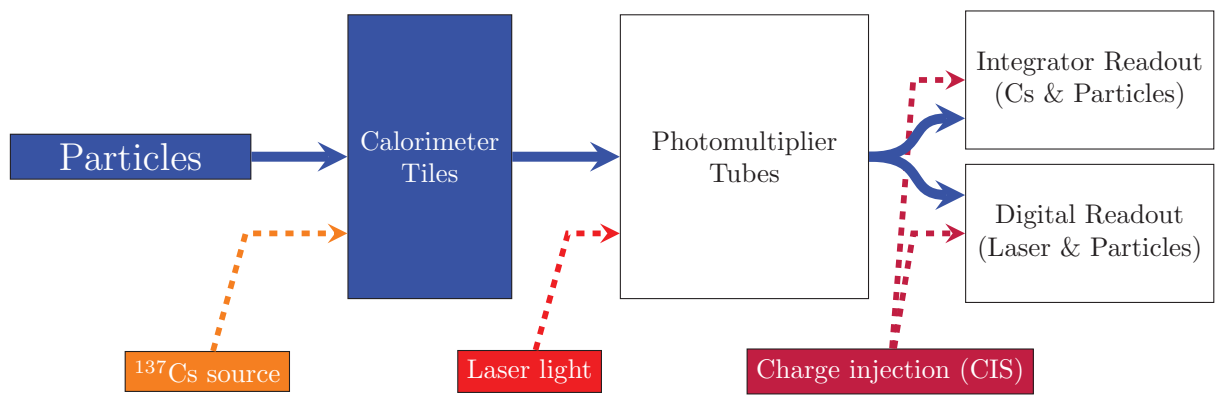

Figure 3. Tile Calorimeter calibration systems chain [3]. Copyright 2017 CERN for the benefit of the ATLAS Collaboration. CC-BY-4.0 license.

weighted sum of samples. With the application of calibration constants, the energy is reconstructed in $\mathrm{GeV}$ units.

To provide correct energy and time information for data reconstruction, an elaborate chain of calibration systems [3], shown in Fig. 3, is used. Charge injection system (CIS) is used to calibrate the response of the ADC. Laser calibration system to measure the performance of the PMTs. Cesium137 radioactive source system $(\mathrm{Cs})$ is used to calibrate the full optical path from scintillating tiles and WLS fibres down to integrated current of the PMT, and a minimum bias monitoring system (MBM) is used to monitor the response of the calorimeter online via integrated currents of PMTs. About $11 \%$ of 192 Tile calorimeter modules were calibrated at the test-beams [4] and the electromagnetic (EM) scale $(1.05 \mathrm{pC} / \mathrm{GeV})$ was transferred to the final detector with the help of calibration systems mentioned above.

\section{Performance}

To reduce the number of non-working ("dead") cells, the on-detector electronics is maintained yearly during detector openings. During the extended year end technical stop (EYETS) of 2016-2017, 48 out of 256 electronics "drawers" were opened and most of the problems were fixed, leading to the $0.06 \%$ of "dead" cells after the stop.

After the restart of the LHC, the increase of the pile-up conditions lead to an increase of the noise levels. The electronics noise is below $20 \mathrm{MeV}$ for the most of the cells, while the pile-up noise increased up to $160 \mathrm{MeV}$ for the inner layer of the calorimeter. 

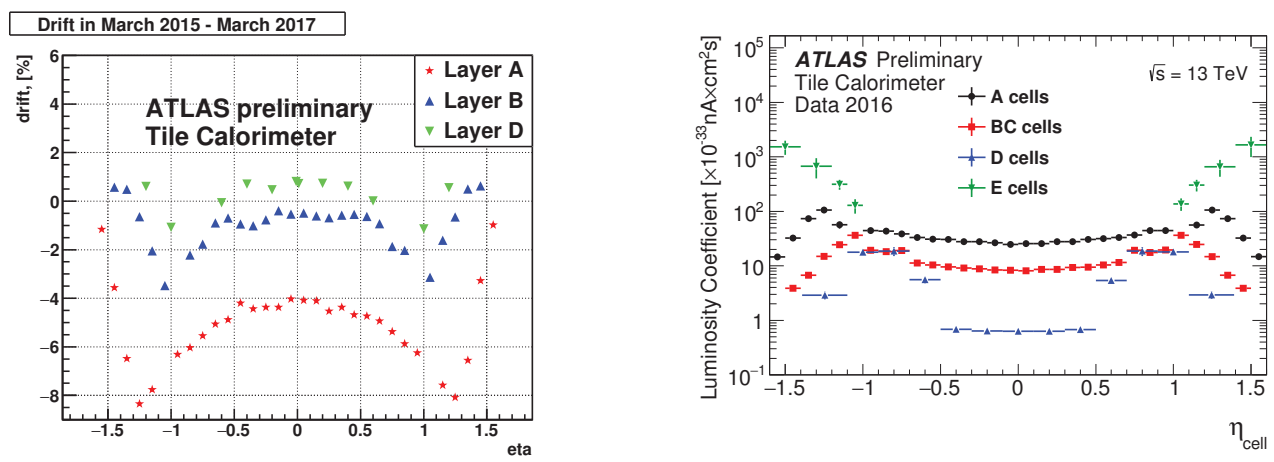

Figure 4. Tile Calorimeter response variation (drift) seen by cesium calibration system vs. cell eta (left) and corresponding cell PMT currents (right) [5]. Copyright 2017 CERN for the benefit of the ATLAS Collaboration. CC-BY-4.0 license.
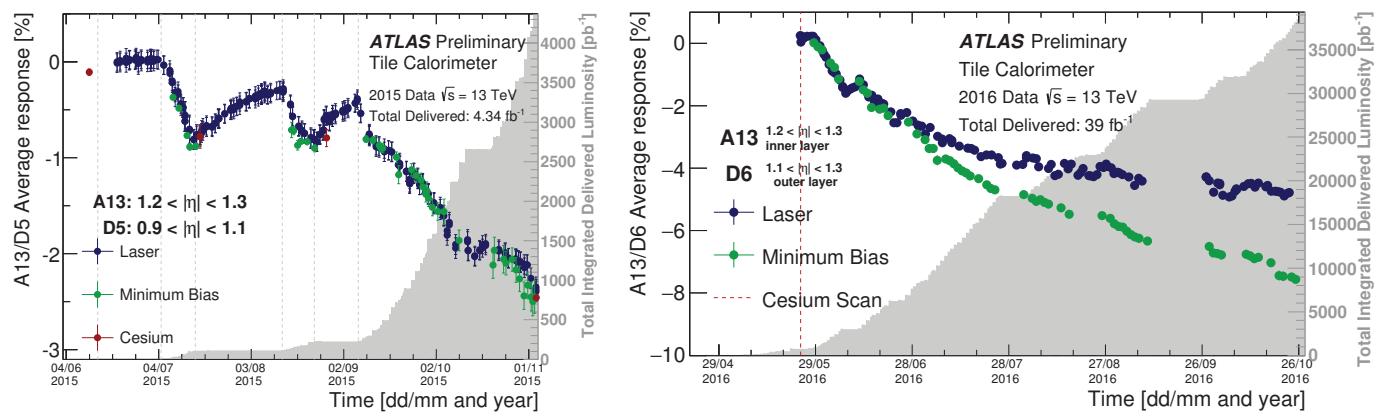

Figure 5. Tile Calorimeter cell response variation in time during 2015 (left) and 2016 (right) pp collisions data taking periods [5]. Copyright 2017 CERN for the benefit of the ATLAS Collaboration. CC-BY-4.0 license.

The cell response is not constant due to the PMT gain variation and scintillator degradation. The response is tracked with the set of calibration systems, described above, as shown in Fig. 4. One of the most exposed cells of the inner calorimeter layer in extended barrel has shown as much as $2 \%$ difference between the laser and integrator response ratios. While the was some recovery during the 2015 data taking, there was practically none during the 2016 data taking period, as shown in Fig. 5. While the PMT response drift can be recovered by raising the high voltage, the loss of the response in the scintillator is taken care of by calibration constants.

One of the important Tile Calorimeter characteristics is the ratio of energy to track momentum $(\mathrm{E} / \mathrm{p})$ for isolated charged hadrons in minimum bias events that it is used to evaluate calorimeter uniformity and linearity during data taking. The data and simulation do agree, showing linearity and uniformity in detector response, and the $\mathrm{dE} / \mathrm{dx}$ of minimum ionising muons show data and MonteCarlo (MC) agreement within 3\%.

Muons from cosmic rays, beam halo and collisions are used to study in-situ the electromagnetic energy scale. A $1 \%$ response non-uniformity in $\eta$ is seen in Tile Calorimeter Long Barrel, with a good energy response uniformity in all calorimeter layers with the data and simulation in agreement. 

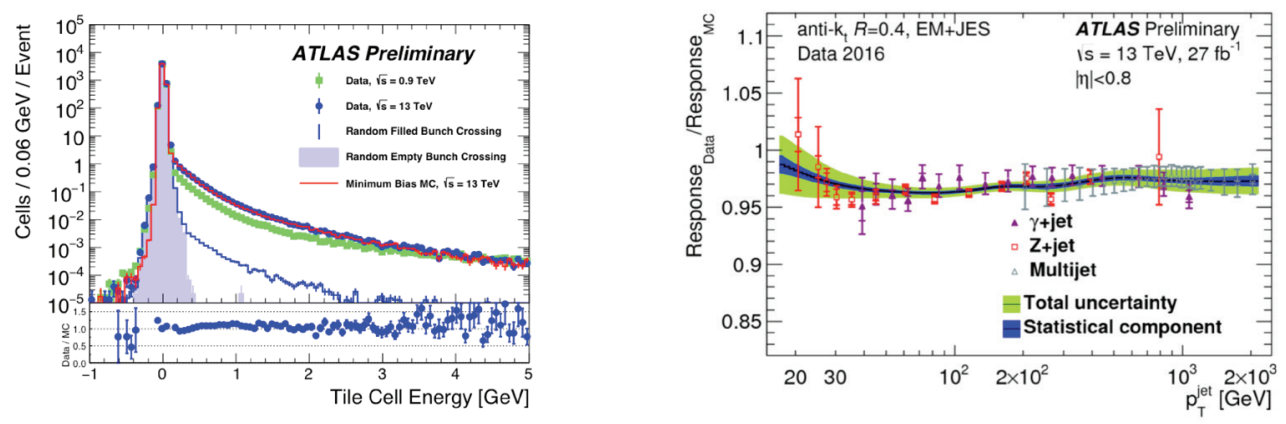

Figure 6. Tile Calorimeter jet performance [5] (left) and ATLAS jet energy data/MC agreement [7] (right). Copyright 2017 CERN for the benefit of the ATLAS Collaboration. CC-BY-4.0 license.

One of the main uses of the hadronic Tile Calorimeter is the measurement of jet energy and missing transverse energy (MET). The data and MC simulations agree well (see Fig. 6), the jet energy scale is consistent [7] with MC predictions, and the jet energy resolution is below $10 \%$ for $\mathrm{p}_{\mathrm{T}}>100 \mathrm{GeV}$. The constant term agree with the expected value of $3 \%$.

The Tile Calorimeter contributes to the ATLAS luminosity measurement by providing a calibration transfer from low to high luminosity conditions, and by longer term luminosity monitoring. Its dedicated readout of the anode currents in every channel is fully decoupled from trigger and is intrinsically independent from pile-up. It also allows a cross-check of other luminometers.

\section{Upgrade}

The upgrade of the LHC machine, the High Luminosity LHC (HL-LHC) will allow almost a factor of 3 increase in integrated luminosity, compared to the simple extension of the running of original accelerator, as it was designed, and almost 10 times more than the data from the first 10 years of LHC operation.

The advanced physics goals and LHC upgrade plans create new challenges for detectors. The upgrade program aims at 5-10 fold luminosity increase, which would mean that more radiation tolerance is required. The ageing electronics, originally planned for 10 years of operation will have to be replaced. Obsolete components will make it impossible to maintain. The high event rates require more efficient trigger algorithms with more primary information from the detectors.

To cope with these issues, a multi-phase upgrade program has been conceived [8]. While the major Tile Calorimeter mechanics and optics will stay, together with associated PMTs. The frontend and back-end electronics, calibration systems, will all undergo major upgrades during Phase-II upgrade stage: complete redesign of the front-end and back-end electronics; a new electronics drawer mechanics; and a fully digital trigger with higher selectivity and finer granularity.

The new readout architecture, shown in Fig. 7, allows the data to be transmitted off-detector at a $40 \mathrm{MHz}$ rate, and to be processed in the off-detector pre-processor boards (PPr). This simplifies the on-detector electronics and provides greater flexibility for the trigger data processing. One of the aims of the upgrade is to improve the redundancy and reliability of the hardware.

The front-end board (FEB) has 3 different options in development [9]. One is based on the well proven original design (3in1) with discrete off-the shelf electronics (see the scheme in Fig. 8), but 


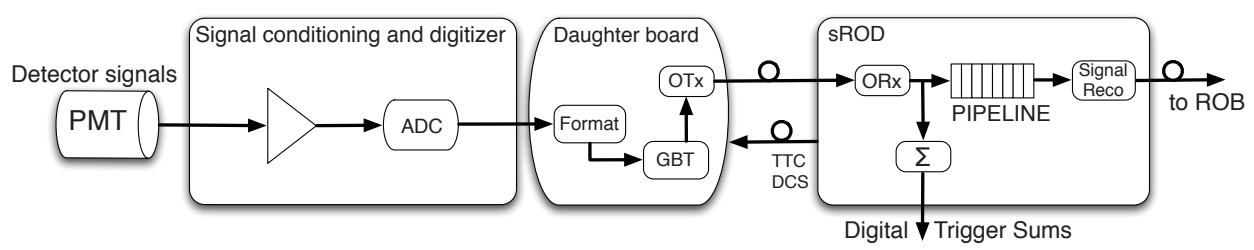

Figure 7. Tile Calorimeter new readout scheme [8]. Copyright 2017 CERN for the benefit of the ATLAS Collaboration. CC-BY-4.0 license.

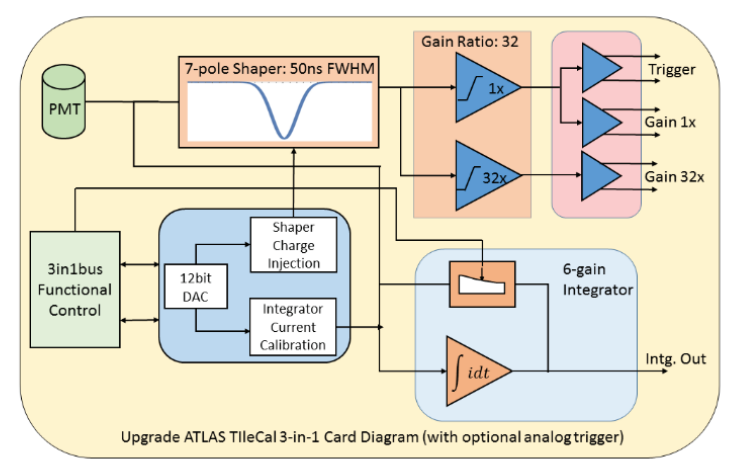

Figure 8. Block diagram of upgraded 3in1 front-end board [9]. Copyright 2017 CERN for the benefit of the ATLAS Collaboration. CC-BY-4.0 license.

with an improved dynamic range and ADC resolution. The other two options (QIE, FATALIC) are based on custom-developed application specific integration circuit (ASIC). Prototypes of all options undergo extensive laboratory and beam tests.

New on-detector electronics requires an upgrade of the low voltage power supply (LVPS). The new design fits the same box, but is simpler, and uses only one voltage level $(10 \mathrm{~V})$, while the required voltages are derived in-situ by point of load regulators (POL). The new powering scheme has also an increased redundancy (see Fig. 9).

The high voltage (HV) distribution system, supplying the detector PMTs, has also to be redesigned. Currently, there are two options. One is based on the original design and regulates the high voltage close to the PMTs (internal option). The so-called remote option [11] would simplify the maintenance and does not require radiation tolerance, a suitable multi-wire high voltage cable is being identified.

To study the performance of different front-end and high-voltage options, a large test-beam campaign is being performed. A set of TileCal modules, equipped both with old and new electronics is being studied with electron, hadron and muon beams, and with calibration systems (charge injection, laser, cesium). 

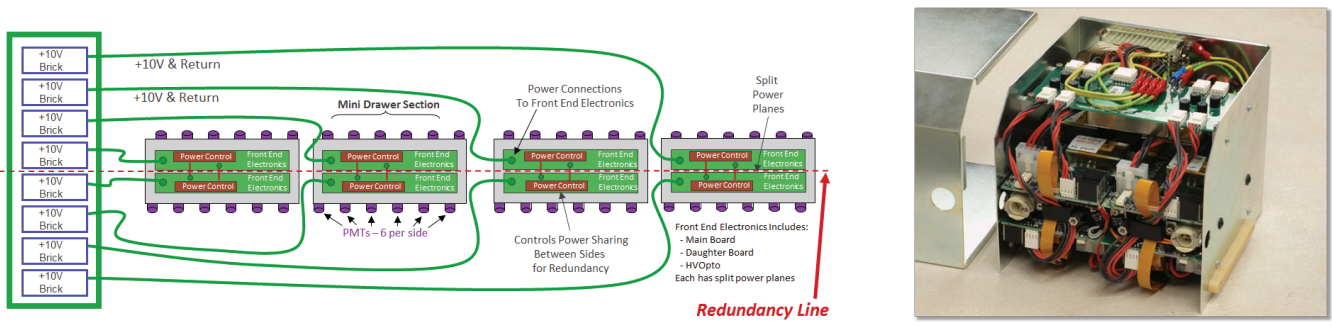

Figure 9. Power distribution scheme of front-end electronics (left) and low voltage power supply box (right) [10]. Copyright 2017 CERN for the benefit of the ATLAS Collaboration. CC-BY-4.0 license.

\section{Summary}

Being an important part of the ATLAS detector at LHC, the Tile Calorimeter has performed very well in LHC Run-2. The calibration systems have allowed the electromagnetic scale to remain within the required precision. The High Luminosity LHC upgrade poses significant challenges and requires a full redesign and replacement of the front-end and back-end electronics. The prototypes of several components are available and are being tested in the labs and at in the test-beam.

\section{References}

[1] ATLAS Collaboration, The ATLAS Experiment at the CERN Large Hadron Collider, JINST 3 (2008) S08003.

[2] ATLAS Collaboration, Readiness of the ATLAS Tile Calorimeter for LHC collisions, Eur. Phys. J. C 70 (2010) 1193.

[3] D. Boumediene et al., Calibration of the Tile Hadronic Calorimeter of ATLAS at LHC, J. Phys. Conf. Ser. 587 (2015) no.1, 012009.

[4] P. Adragna et al., Testbeam studies of production modules of the ATLAS tile calorimeter, Nucl. Instrum. Meth. A 606 (2009) 362.

[5] ATLAS Tile Calorimeter Subsystem, Approved Plots, Accessed 20 September 2017. https://twiki.cern.ch/twiki/bin/view/AtlasPublic/ApprovedPlotsTile.

[6] K. Anderson et al., Design of the front-end analog electronics for the ATLAS tile calorimeter, Nucl. Instrum. Meth. A 551 (2005) 469.

[7] M. Aaboud et al. [ATLAS Collaboration], Jet energy scale measurements and their systematic uncertainties in proton-proton collisions at $\sqrt{s}=13 \mathrm{TeV}$ with the ATLAS detector, CERN-EP2017-038, arXiv:1703.09665 [hep-ex].

[8] G. Usai et al., ATLAS Tile Calorimeter electronics and future upgrades, JINST 10 (2015) no.04, C04026.

[9] A. Gomes et al., The new front-end electronics for the ATLAS Tile Calorimeter Phase 2 Upgrade, JINST 11 (2016) no.02, C02015.

[10] A. Solodkov et al., Upgrade of the ATLAS hadronic Tile Calorimeter for the High luminosity LHC, JINST 12 (2017) no.08, C08004.

[11] F. Vazeille et al., Performance of a remote High Voltage power supply for the Phase II upgrade of the ATLAS Tile Calorimeter, JINST 11 (2016) no.02, C02050. 